\title{
Dinámica de la dieta de Asplanchna girodi (De Guerne, 1888) en un embalse tropical, Colombia
}

\author{
The dynamics of the diet of Asplanchna girodi (De Guerne, 1888) \\ inhabiting a tropical reservoir in Colombia
}

\section{Dinâmica da dieta de Asplanchna girodi (De Guerne, 1888) em um reservatório tropical, Colômbia}

\author{
Ana L Estrada-Posada ${ }^{*}$; Miguel A Giraldo-Moy ${ }^{2}$ * \\ 1 Bióloga MSc, \\ 2 Biólogo \\ * Instituto de Biología, Facultad de Ciencias Exactas y Naturales, Universidad de Antioquia, Colombia \\ Email: limnobio.2007@gmail.com
}

Recibido: junio 6 de 2018

Aceptado: diciembre 6 de 2018

\begin{abstract}
Resumen
Las interacciones bióticas como depredación, forrajeo y herbivoría han sido generalmente catalogadas como las fuerzas que determinan las variaciones espaciales y temporales de las poblaciones planctónicas, tanto de presas como de depredadores. Una especie del zooplancton, presente en aguas continentales, calificada como depredadora es el rotífero Asplanchna girodi. Sin embargo, se desconoce la composición real de su dieta, las preferencias alimentarias y la dinámica espacial y temporal en relación con sus presas o alimento. En esta investigación se determinaron las preferencias tróficas de $A$. girodi y su fluctuación espacio-temporal, basados en ítems verdaderamente consumidos en el medio natural, a través del análisis de los contenidos estomacales de individuos capturados en las aguas del embalse Riogrande II (Antioquia-Colombia), durante un año de muestreos realizados cada 10 días. Se observó que A. girodi presentó una preferencia trófica amplia que incluyó el forrajeo de, por lo menos, cinco taxones de algas y la depredación de ocho (8) especies de rotíferos y del cladócero Bosmina freyi. Se concluye que: 1 . A. girodi es una especie omnívora, con mayor preferencia por el consumo de algas. 2. Este rotífero no fue factor de presión en la dinámica poblacional de las algas, pero posiblemente lo fue para Keratella americana. 3. No se observaron diferencias espaciales en la dieta de $A$. girodi, pero si existieron diferencias temporales en cuanto a la composición de la dieta, la cual estuvo determinada por la oferta de las algas, principalmente durante florecimientos de Ceratium. 4. Este tipo de dieta se enmarca dentro de los conceptos de forrajeo óptimo y oportunismo trófico, estrategias que conllevan el consumo de las especies con mayor disponibilidad (en este caso algas) y el máximo ahorro de energía, puesto que no se invierte en la búsqueda de presas específicas.
\end{abstract}

Palabras clave: Asplanchna; forrajeo óptimo; oportunismo trófico; Riogrande II; rotíferos.

\begin{abstract}
Biotic interactions such as predation, foraging and herbivory have been generally categorized as the forces that determine the spatial and temporal variations of planktonic populations, both preys and predators. A species of zooplankton, present in continental waters, classified as predator is the rotifer Asplanchna girodi. However, the true composition of its diet, dietary preferences and spatial and temporal dynamics in relation to its prey or food are unknown. In this research the
\end{abstract}


trophic preferences of $A$. girodi and its spatio-temporal fluctuation were determined, based on items truly consumed in the natural environment, through analysis of stomach contents of individuals captured in the waters of Riogrande II reservoir (Antioquia-Colombia), during one year of sampling every 10 days. It was observed that $A$. girodi showed a broad trophic preference that included the foraging of, at least, five algae taxa and the predation of eight rotifer species and cladoceran Bosmina freyi. It is concluded that: 1 . A. girodi is an omnivorous species, with a greater preference for the consumption of algae. 2. This rotifer was not a pressure factor in the population dynamics of the algae, but possibly it was for the Keratella americana. 3. No spatial differences were observed in the A. girodi diet, but there were temporal differences in the composition of the diet, which was determined by the algae supply, mainly during Ceratium blooms. 4. This type of diet is framed within the concepts of optimal foraging and trophic opportunism, strategies that involve the consumption of the most available species (in this case algae) and the maximum energy saving since it is not invested in the search for specific prey.

Key words: Asplanchna; optimal foraging; trophic opportunism; Riogrande II; rotifers.

\section{Resumo}

Interações bióticas como predação, forrageamento e herbivoria têm sido geralmente classificadas como as forças que determinam as variações espaciais e temporais de populações planctônicas, tanto de presas quanto de predadores. Uma espécie de zooplâncton, presente em águas continentais, classificada como predador é o rotífero Asplanchna girodi. No entanto, a composição real de sua dieta, preferências alimentares e dinâmicas espaciais e temporais em relação a suas presas ou alimentos são desconhecidas. Nesta pesquisa, as preferências tróficas de A. girodi e sua flutuação espaçotemporal foram determinadas, com base em itens verdadeiramente consumidos no ambiente natural, através da análise do conteúdo estomacal de indivíduos capturados nas águas do reservatório Riogrande II (Antioquia-Colômbia), durante um ano de amostragem a cada 10 dias. Observou-se que A. girodi apresentou uma ampla preferência trófica que incluiu o forrageamento de pelo menos cinco taxa de algas e a predação de oito (8) espécies de rotíferos e o cladocero Bosmina freyi. Conclui-se que: 1. A. girodi é uma espécie onívora, com maior preferência pelo consumo de algas. 2. Este rotífero não foi um fator de pressão na dinâmica populacional das algas, mas possivelmente foi para Keratella americana. 3. Não foram observadas diferenças espaciais na dieta de A. girodi, mas houve diferenças temporárias na composição da dieta, que foi determinado pelo fornecimento de algas, principalmente durante a floração de Ceratium. Este tipo de dieta enquadra-se nos conceitos de forrageamento ótimo e oportunismo trófico; estratégias que envolvem o consumo de espécies com maior disponibilidade (neste caso algas) e a máxima economia de energia, uma vez que não é investido na busca por presas específicas.

Palavras-chave: Asplanchna; forrageamento ótimo; oportunismo trófico; Riogrande II; rotíferos

\section{Introducción}

Las interacciones bióticas como la depredación, el forrajeo o herbivoría y la disponibilidad del alimento han sido generalmente catalogadas como las fuerzas que determinan las variaciones espaciales y temporales del zooplancton (Lampert y Sommer, 1997). Los depredadores y forrajeros pueden ser controladores de la abundancia y de la presencia de diferentes organismos en los ecosistemas acuáticos; o contrastantemente estos mismos pueden ajustarse espacial y temporalmente según la oferta trófica (Begon et al., 2006, Da Silva y Hahn, 2009).

Dentro del zooplancton algunos géneros han sido descritos como depredadores, entre ellos, las publicaciones refieren a Asplanchna como un género omnívoro que preferiblemente se alimenta de otros rotíferos y cladóceros pequeños (Conde-Porcuna et al., 1993; Gilbert y Jack 1993; Sarma y Dumont 1995, Kappes et al., 2000); con diferencias en la composición de las dietas según la especie (Chang et al., 2010).

Algunos de estos estudios han establecido que la dieta de Asplanchna está limitada por el tamaño, morfología, densidad, estructuras defensivas y capacidad evasiva de sus presas. No obstante lo anterior, varios estudios han demostrado que una vez las densidades de algunas presas decaen, el rotífero Asplanchna cambia su dieta a otras especies más abundantes (Conde Porcuna et al., 1993, Sarma et al., 1998).

Chang y colaboradores (2010) han reportado la inclusión de algas en la dieta de Asplanchna, como es el caso para A. priodonta y A. herricki; sin embargo, en este estudio los autores indican que la dieta de A. girodi está compuesta en su mayoría por especies del zooplancton, catalogándola como depredadora.

Los estudios realizados hasta el momento han mencionado algunos elementos que identifican las preferencias alimenticias del zooplancton; sin embargo, estos trabajos acotan detalles del comportamiento del zooplancton en experimentos de laboratorio controlados que utilizan presas y depredadores específicos, pero que han ignorado la fluctuación en la dieta de las poblaciones naturales de acuerdo con la disponibilidad de los diferentes ítems alimenticios y la presencia de depredadores diversos en el escenario real. Este tipo de estudios han reforzado el concepto de cadena tró- 
fica (Van der Stap et al., 2007), y han rezagado el concepto de la red trófica, por tanto, las conclusiones han sido unidireccionales y han ignorado la existencia de depredadores y herbívoros poco selectivos y presas de baja especificidad.

Puesto que el género Asplanchna ha sido tipificado como un depredador, la realización de experimentos de laboratorio en espacios confinados se ha limitado al uso de presas de rotíferos (Sarma y Nandini, 2007), pero en pocas oportunidades se han incluido algas de diferentes especies. Lo anterior ha arrojado conclusiones sesgadas sobre las preferencias alimenticias y la energía empleada por Asplanchna para capturar las especies de preferencia en el medio natural (Chang et al., 2010).

Una especie de Asplanchna de amplia distribución en las aguas continentales es A. girodi. Esta es considerada como un depredador para el cual se desconoce la dieta, las preferencias alimentarias y la dinámica espacial y temporal en relación con sus presas o alimento en general. En la presente investigación se pretendió determinar las preferencias tróficas del rotífero A. girodi y su fluctuación espacio-temporal, basados en ítems realmente consumidos en el medio natural a través del análisis de los contenidos estomacales de individuos de esta especie; capturados en las aguas del embalse Riogrande II (Antioquia-Colombia), durante un año de muestreos.

En esta investigación se plantearon tres hipótesis:
Hipótesis 1: Si los contenidos estomacales de A. girodi muestran una composición heterogénea de ítems alimenticios (tanto fito como zooplancton) entonces $A$. girodi será una especie omnívora, con tendencia hacia la herbivoría, dado que el consumo de algas requiere menor inversión energética.

Hipótesis 2: Si las preferencias alimenticias de A. girodi constituyen un factor de presión en la dinámica poblacional de las algas y/o zoopláncteres consumidos, entonces existe una relación significativa de $A$. girodi con la distribución y densidad de los ítems forrajeados en el embalse Riogrande II.

Hipótesis 3: Si la dieta y densidad de A. girodi fluctúa en el espacio y el tiempo, entonces la presencia y densidad de sus ítems alimenticios más habituales determinan la dinámica espacio-temporal de este rotífero en el embalse Riogrande II.

\section{Materiales y métodos}

\section{Descripción del área de estudio}

El embalse Riogrande II está localizado en la parte central del departamento de Antioquia (Colombia), en la cuenca del río Grande (6을 $35^{\prime} 57^{\prime \prime}-6^{\circ} 28^{\prime} 79^{\prime \prime} \mathrm{N}$ y los $75^{\circ} 32^{\prime} 17^{\prime \prime}$ y $75^{\circ} 25^{\prime} 8^{\prime \prime}$ O). La cuenca aportante tiene una extensión de 1214 hectáreas y la conforman los municipios de Donmatías, Entrerríos, San Pedro de Los Milagros y Santa Rosa de Osos. En el embalse Riogrande II se represan las aguas aportadas por las subcuen-

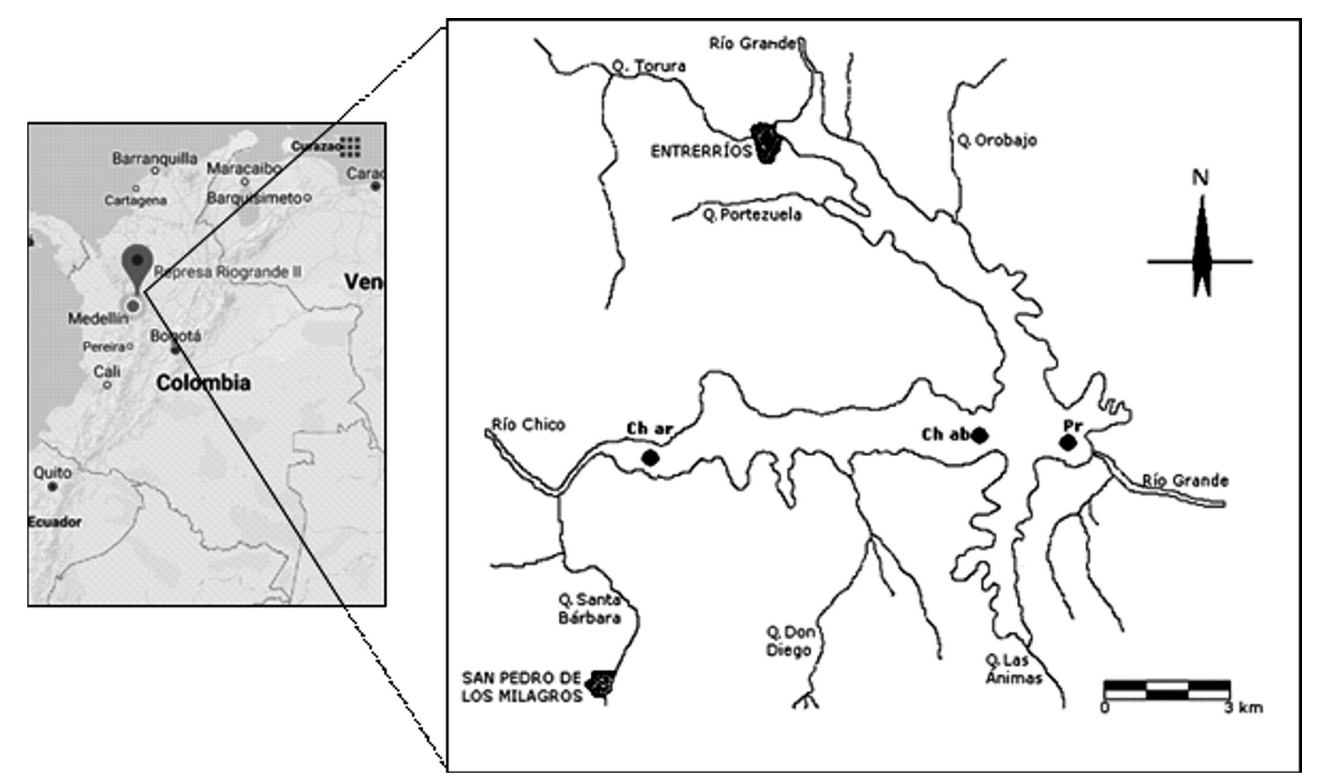

Figura 1. Sitios de muestreo en el eje del río Chico, embalse Riogrande II (Ch ar: Chico arriba, Ch ab: Chico abajo y Pr: Presa). Fuentes: Google maps (2018), Giraldo (2007). 
cas del río Grande y del río Chico y la quebrada Las Ánimas (Mazo et al., 2015) (Figura 1).

El llenado del vaso inició en noviembre de 1990. La cota máxima del embalse está ubicada a 2270 msnm, con un volumen total de 240.58 millones de $\mathrm{m}^{3}$, profundidad máxima de $42.0 \mathrm{~m}$ y media de $37.9 \mathrm{~m}$. El tiempo de residencia es de 72.8 días, la longitud del brazo más largo (río Chico) es de $10 \mathrm{~km}$. Los caudales anuales promedio del río Chico son de $4.7 \mathrm{~m}^{3} / \mathrm{s}(1.5$ $\left.12.0 \mathrm{~m}^{3} / \mathrm{s}\right)$ y del río Grande de $29.0 \mathrm{~m}^{3} / \mathrm{s}(16.7$ - 43.8 $\mathrm{m}^{3} / \mathrm{s}$ ). La cuenca sobre la cual está asentado el embalse presenta dos periodos lluviosos abril-mayo y septiembre-octubre. La precipitación anual en la cuenca oscila entre los 1800 y $3000 \mathrm{~mm}$, con un rendimiento hídrico promedio de $19 \mathrm{l} / \mathrm{s} / \mathrm{km}^{2}$ (EPM 2005, Giraldo, 2010).

El aprovechamiento múltiple del embalse incluye la generación hidroeléctrica, el mejoramiento ambiental y el abastecimiento del sistema de acueducto de la ciudad de Medellín y los municipios de Bello, Itagüí, Envigado y Sabaneta (EPM 2005).

\section{Evaluación de variables abióticas}

Esta investigación empleó la base de datos obtenida por Estrada-Posada (2007) entre agosto de 2002 y julio de 2003; con muestreos realizados cada diez días en tres sitios de colecta a lo largo del eje longitudinal del río Chico en el embalse Riogrande II, a saber:

- Río Chico arriba: 6³0'37" N 75³2'17" O. Cola embalse, brazo río Chico.

- Río Chico abajo: 6³0'66" N 75²8'39" O. Zona Media, brazo río Chico.

- Presa: $6^{\circ} 30^{\prime} 25^{\prime \prime} \mathrm{N} 75^{\circ} 27^{\prime} 01^{\prime \prime} \mathrm{O}$. A $0.5 \mathrm{~km}$ de la presa

En estos sitios se colectaron muestras de agua para evaluar variables físicas, químicas y biológicas, algunas de las cuales fueron medidas en campo y las restantes fueron cuantificadas en los laboratorios de Ingeniería Sanitaria y de Limnología (Grupo LimnoBasE BiotaMar) de la Universidad de Antioquia.

En la columna de agua se realizaron perfiles verticales de temperatura y oxígeno disuelto cada $25 \mathrm{~cm}$ en los primeros diez metros, y cada metro hasta el fondo. Además, se realizaron perfiles de conductividad eléctrica, $\mathrm{pH}$, alcalinidad total y turbidez, cada $5 \mathrm{~m}$ en los sitios más profundos, y cada $2.5 \mathrm{~m}$ en el sitio Chico arriba. Dentro de la zona fótica se colectaron muestras de agua a tres profundidades diferentes y se integraron para evaluar las concentraciones de sólidos totales, sólidos disueltos, sólidos suspendidos totales, sólidos volátiles y sólidos fijos, fósforo soluble, nitratos, amonios, nitrógeno total y demanda química de oxígeno. Los métodos para analizar las variables mencionadas se registraron en Estrada-Posada (2007).

\section{Evaluación de variables bióticas}

Para la colecta del zooplancton se integraron cuatro profundidades dentro de la zona fótica de cada sitio de muestreo, tomando un total de 20 litros con una botella Schindler; de estos se extrajeron $500 \mathrm{ml}$ para evaluar la biomasa fitoplanctónica y el volumen restante se filtró en un tamiz con poro de $60 \mu \mathrm{m}$, concentrándose la muestra en un vial de $50 \mathrm{ml}$ para determinar la composición y densidad del zooplancton.

Las muestras para evaluar la biomasa fitoplanctónica fueron almacenadas en botellas oscuras a $4{ }^{\circ} \mathrm{C}$; el zooplancton fue conservado en frío y horas después fijado con una mezcla de azúcar y solución transeau (formol al $37 \%$, alcohol al $96 \%$ y muestra de agua, en proporciones 1: 3: 6) para controlar deformaciones (Haney y Hall, 1973).

La biomasa fitoplanctónica se estimó mediante el método de la clorofila a, filtrando un volumen de agua conocido a través de un filtro de celulosa $(\varnothing=45 \mu \mathrm{m})$, y utilizando como solvente extractor etanol caliente al $96 \%$. Para discriminar entre clorofila a y feopigmentos se acidificó el extracto con $\mathrm{HCl} 0.01$ N (Sartory y Grobbelaar, 1984).

La concentración de clorofila a se estimó con la siguiente ecuación:

Clorofila a= 29.14 (A665-750 - A665a-750a) x v/ V x I ( $\mu \mathrm{g} / \mathrm{l})$.

A665-750: absorbancia de la muestra sin acidificar

A665a-750a: absorbancia de la muestra después de acidificar

v: volumen utilizado de etanol, en mililitros

V: volumen filtrado de muestra, en litros

I: longitud de paso de la celda del espectrofotómetro, en centímetros

Considerando que el estudio de los contenidos estomacales es la manera más adecuada de investigar el papel de los alimentos dentro de la comunidad de interés (Hyslop 1980), se identificaron los ítems o taxones que constituyeron la dieta del rotífero $A$. girodi, en cada fecha y sitio de muestreo mediante el análisis de los contenidos estomacales de 30 hembras selecciona- 
das al azar, por medio de la digestión progresiva de las partes blandas del cuerpo con hipoclorito. Se revisaron aproximadamente 2610 ejemplares o estómagos.

Previo a la determinación taxonómica de los ítems alimenticios presentes en los contenidos estomacales, se realizó la identificación de todas las especies que componían el fito y el zooplancton del embalse Riogrande II; lo que implicó el estudio de las características de los trofi de todas las especies de los rotíferos presentes y las partes que componían a otras especies del plancton, incluidas las algas.

La proporción de cada ítem en la dieta de $A$. girodi se estimó mediante la integración de los contenidos de los 30 estómagos analizados por muestra. La importancia de cada ítem alimentario del grupo del zooplancton fue evaluada mediante el Índice de Importancia Alimentaria modificado y propuesto por Lauzanne (1975) así:

$\mathrm{IAA}=(\mathrm{FOi} \times \mathrm{Ni}) / 100$

FOi: Frecuencia de ocurrencia del ítem alimentario i

Ni: número de individuos i encontrados en el contenido estomacal

Mediante este índice se estableció la selectividad del alimento y cuatro categorías de importancia: presa dominante: $>50$, presa esencial: 25-50, presa de importancia moderada: $10-25$ y presa secundaria: $<10$.

El Índice de Importancia Alimentaria sólo se aplicó para el zooplancton, porque en este grupo se pudo realizar el análisis cuantitativo, ya que se encontraban los organismos completos o los trofi (cada trofi corresponde a un individuo), mientras que con el fitoplancton no fue posible establecer una valoración cuantitativa, dado que sólo se encontraban partes o segmentos que no daban cuenta de la cantidad de individuos consumidos.

Una vez identificadas las especies que formaron parte de la dieta de $A$. girodi se cuantificaron las densidades poblacionales de éstas y de $A$. girodi, con el fin de determinar la dinámica de la distribución espacial y temporal. El número de organismos y el volumen filtrado fueron empleados para estimar la densidad de las poblaciones por litro.

La determinación taxonómica de los rotíferos, cladóceros y copépodos se realizó usando las claves taxonómicas de Edmonson (1965), Rocha y Tundisi (1976), Koste (1978), Sendacz y Kubo (1982), Reid (1985), Koste y Shield (1987), Pennak (1989), Shield y Koste (1992, 1993), Gaviria (1994), Fernando (2002), Segers
(2003), Elmoor-Loureiro et al., (2004). Para la determinación taxonómica de las algas se usaron las claves de Prescott (1962), Bicudo y Bicudo (1970), Ramírez (2000), Jena y Adhikary (2007).

\section{Análisis estadístico}

Se realizó análisis estadístico descriptivo de los datos obtenidos de las variables abióticas y bióticas, mediante el uso de media aritmética, mediana, desviación estándar y coeficiente de variación de Pearson.

Para determinar la existencia de diferencias con significancia estadística entre los sitios de muestreo -variación horizontal- y entre las fechas -variación temporal- de acuerdo con las variables abióticas y bióticas, se emplearon las pruebas de Análisis de varianza (Múltiple Sample Comparison) y de Tukey, para comparar medias en distribuciones que cumplieron con los supuestos exigidos por la estadística paramétrica. Las pruebas Kruskal-Wallis y de Mann-Whitney se usaron para comparar las medianas en aquellas variables que después de haberlas sometido a diferentes transformaciones no cumplieron con las exigencias de los análisis paramétricos.

Además, se empleó un análisis multivariante de discriminantes, para clasificar las fechas y sitios de muestreo, en función de los ítems alimenticios. Se empleó inicialmente un análisis exploratorio de componentes principales para escoger los taxones frecuentes y descartar los raros (que aportaron poco a la varianza).

Puesto que los datos no cumplieron con los requisitos de la estadística paramétrica, se aplicó el coeficiente de correlación de Spearman para comprobar si existía asociación entre las densidades de los diferentes taxones del zooplancton, el componente abiótico, la clorofila a, y la densidad total del zooplancton.

La dependencia o relación de las densidades poblacionales entre $A$. girodi y sus presas, en el tiempo y el espacio se analizaron mediante análisis de correlación de Spearman y regresión lineal, usando los datos de las densidades de cada especie. Para los análisis anteriores se emplearon los programas STATISTICA 6.0, STATGRAPHICS Centurion y CANOCO 3.5.

\section{Resultados}

Las características abióticas evaluadas mostraron heterogeneidad espacial en el eje longitudinal del río Chico, con mayor contraste entre los sitios Chico arriba y Presa (Tabla 1). El sitio Chico arriba se diferencia de los otros sitios, por estar ubicado en una de las colas del 
Tabla 1. Tendencia central y de dispersión de las variables ambientales evaluadas en la zona fótica que presentaron diferencias espaciales (resultados pruebas ANOVA y ad hocTukey o de U), en tres sitios de muestreo en el embalse Riogrande II, agosto 2002 - julio 2003.

\begin{tabular}{|c|c|c|c|c|c|}
\hline \multirow{2}{*}{$\begin{array}{c}\text { Variable } \\
\text { Unidad. Tukey - U }\end{array}$} & \multirow{2}{*}{$\begin{array}{l}\text { Grados de } \\
\text { libertad }\end{array}$} & \multirow{2}{*}{ Estadístico } & \multicolumn{3}{|c|}{ Sitios de muestreo } \\
\hline & & & Ch arriba & Ch abajo & Presa \\
\hline \multirow{2}{*}{$\begin{array}{l}\text { Profundidad máxima } \\
(\mathrm{m}) . \mathrm{F}=83.41, \mathrm{p}=<0.01\end{array}$} & \multirow{2}{*}{35} & Promedio & 13.13 & 26.53 & 35.52 \\
\hline & & CV (\%) & 22.41 & 24.24 & 17.00 \\
\hline \multirow{2}{*}{$\begin{array}{l}\text { Extensión Zona Fótica } \\
(\mathrm{m}) . \mathrm{F}=11.10, \mathrm{p}=<0.01\end{array}$} & \multirow{2}{*}{35} & Promedio & 3.37 & 5.53 & 5.06 \\
\hline & & CV $(\%)$ & 43.12 & 31.69 & 29.71 \\
\hline \multirow{2}{*}{$\begin{array}{l}\text { Temperatura del agua } \\
\left({ }^{\circ} \mathrm{C}\right) . \mathrm{F}=6.60, p=<0.01\end{array}$} & \multirow{2}{*}{35} & Promedio & 22.6 & 21.3 & 20.7 \\
\hline & & CV $(\%)$ & 4.5 & 3.2 & 4.66 \\
\hline \multirow{2}{*}{$\begin{array}{l}\text { Oxígeno Disuelto } \\
(\mathrm{mg} / \mathrm{l}) . \mathrm{X}^{2}=22.75, \mathrm{p}=<0.01\end{array}$} & \multirow{2}{*}{35} & Promedio & 8.00 & 7.15 & 6.72 \\
\hline & & CV (\%) & 18.93 & 12.32 & 22.40 \\
\hline \multirow{2}{*}{$\begin{array}{l}\text { Conductividad Eléctrica } \\
\text { (uS/cm). } \mathrm{F}=16.32, \mathrm{p}=<0.01\end{array}$} & \multirow{2}{*}{35} & Promedio & 47.6 & 38.9 & 36.3 \\
\hline & & CV $(\%)$ & 9.41 & 9.50 & 11.00 \\
\hline \multirow{2}{*}{$\begin{array}{l}\mathrm{pH} \text { (Unidades). } \\
\mathrm{X}^{2}=49.63, \mathrm{p}=<0.01\end{array}$} & \multirow{2}{*}{35} & Promedio & 9.61 & 9.11 & 8.92 \\
\hline & & CV (\%) & 3.70 & 6.44 & 6.56 \\
\hline \multirow{2}{*}{$\begin{array}{l}\text { Turbidez } \\
\text { (FTU). } X^{2}=19.49, p=<0.01\end{array}$} & \multirow{2}{*}{35} & Promedio & 28.55 & 9.31 & 10.55 \\
\hline & & $\mathrm{CV}(\%)$ & 257.30 & 57.48 & 66.51 \\
\hline \multirow{2}{*}{$\begin{array}{l}\text { Sólidos Totales } \\
(\mathrm{mg} / \mathrm{l}) . \mathrm{X}^{2}=19.04, \mathrm{p}=<0.01\end{array}$} & \multirow{2}{*}{35} & Promedio & 80.6 & 68.6 & 64.8 \\
\hline & & CV $(\%)$ & 24.40 & 20.26 & 20.79 \\
\hline \multirow{2}{*}{$\begin{array}{l}\text { Sólidos Suspendidos Total } \\
(\mathrm{mg} / \mathrm{l}) . \mathrm{X}^{2}=27.06, \mathrm{p}=<0.01\end{array}$} & \multirow{2}{*}{35} & Promedio & 16.23 & 7.36 & 7.47 \\
\hline & & $\mathrm{CV}(\%)$ & 102.63 & 31.76 & 33.92 \\
\hline \multirow{2}{*}{$\begin{array}{l}\text { Sólidos Volátiles Totales } \\
(\mathrm{mg} / \mathrm{l}) . \mathrm{F}=7.17, \mathrm{p}=<0.01\end{array}$} & \multirow{2}{*}{35} & Promedio & 53.2 & 43.4 & 37.8 \\
\hline & & CV $(\%)$ & 27.45 & 30.14 & 30.57 \\
\hline \multirow{2}{*}{$\begin{array}{l}\text { Sólidos Disueltos } \\
(\mathrm{mg} / \mathrm{l}) . \mathrm{X}^{2}=10.19, \mathrm{p}=<0.05\end{array}$} & \multirow{2}{*}{35} & Promedio & 64.33 & 61.25 & 57.32 \\
\hline & & CV (\%) & 22.44 & 22.47 & 22.14 \\
\hline \multirow{2}{*}{$\begin{array}{l}\text { Fósforo soluble reactivo } \\
(\mathrm{mg} / \mathrm{l}) . \mathrm{F}=6.24, \mathrm{p}=<0.01\end{array}$} & \multirow{2}{*}{11} & Promedio & 0.14 & 0.07 & 0.04 \\
\hline & & CV (\%) & 54.70 & 129.88 & 218.53 \\
\hline
\end{tabular}

embalse, y se caracterizó por presentar menor profundidad y zona fótica estrecha, y mayores valores de oxígeno disuelto, conductividad eléctrica, $\mathrm{pH}$, turbidez, sólidos y fósforo soluble reactivo.

Las concentraciones de clorofila en el sitio Chico arriba fueron mayores (106.56 ug/l en promedio) y comparativamente bajas en Chico abajo y Presa (25.41 y $18.40 \mathrm{ug} / \mathrm{l}$, respectivamente). En el embalse Riogrande Il los diez taxones de fitoplancton con mayores densidades para el año de estudio en orden descendente fueron: Staurastrum, Chlorococcales, Chlamydomonas, Schroederia, Monoraphidium, Dolichospermum, Cera- tium, Cryptomonas, diatomeas y Nephrocytium (Figura 2). Las densidades promedio de estos taxones oscilaron entre 94 (Nephrocytium) y $1384 \mathrm{ind} / \mathrm{ml}$ (Staurastrum) (Estrada-Posada, 2007).

En relación con el zooplancton se registraron 24 especies en los tres sitios de estudio, pertenecientes a los grupos Ciliophora (Campanella umbellaria); Rotifera (Anuraeopsis navicula, Ascomorphella volvocicola, Asplanchna girodi, A. sieboldi, Collotheca trilobata, Filinia longiseta, Hexarthra intermedia, Keratella americana, $K$. cochlearis, K. tropica, Polyarthra vulgaris, Ptygura libera, Synchaeta pectinata, Trichocerca sp, T. cylindrica, T. 


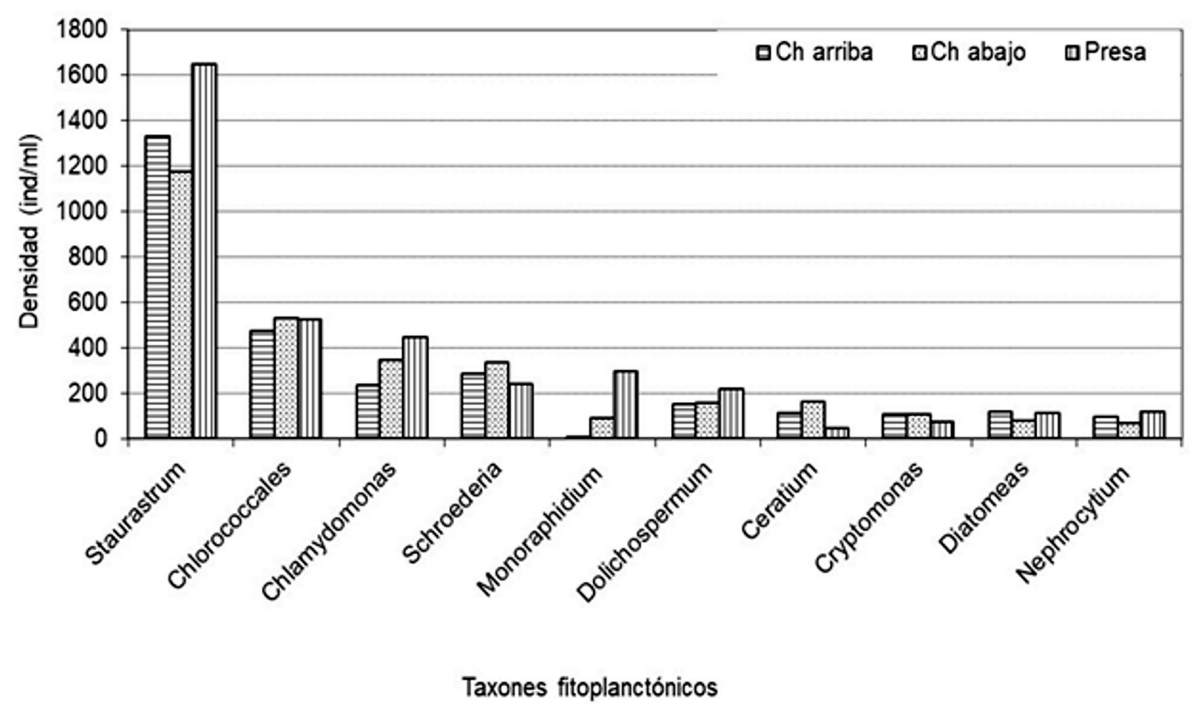

Figura 2. Estructura del fitoplancton con mayores densidades, en los tres sitios de muestreo del embalse Riogrande II, en el periodo agosto 2002 - julio 2003.

similis, T. stylata); Cladocera (Bosmina freyi, Daphnia ambigua, Diaphanosoma birgei, Moina micrura) y $\mathrm{Co}-$ pepoda (Tropocyclops prasinus, Mesocyclops longisetus).

Los taxones del zooplancton con mayores densidades para el período de estudio, en orden descendente fueron: C. umbellaria (Linnaeus, 1767), B. freyi (De Melo y Hebert, 1994), A. girodi, (De Guerne, 1888), P. vulgaris (Carlin, 1943), H. intermedia (Hauer, 1953), T. similis (Wierzejski, 1893) y los nauplios de T. prasinus (Fischer, 1860). En el embalse la riqueza varió entre 12 y 19 especies por muestreo. El sitio Chico arriba presentó mayor fluctuación en la riqueza (entre 6 y 17 especies) (Estrada-Posada, 2007).

El rotífero A. girodi y cinco (5) de las especies del zooplancton registradas en los contenidos estomacales presentaron disposición longitudinal heterogénea (Tabla 2), reiterándose al sitio Chico arriba como diferente de los demás con un $\mathrm{P}<0.05$. Las especies que mostraron heterogeneidad longitudinal fueron: $B$. freyi (Cladocera), y los rotíferos $T$. similis, $P$. vulgaris, $H$. intermedia y F. longiseta. En cuanto a la dimensión temporal se observó aparición esporádica del género Keratella.

Los análisis de los contenidos estomacales de aproximadamente 2610 individuos de A. girodi, colectados en el eje del río Chico, mostraron que la dieta de este rotífero incluye dos grandes grupos: por lo menos cinco taxones del fitoplancton y nueve taxones del zooplancton (Tabla 3).

Para el Orden Chlorococcales y los demás géneros del fitoplancton la identificación hasta especie dentro de los contenidos estomacales no fue posible, dado que se encontraban tan sólo partes de los organismos. Sin embargo, algunos de los géneros en el embalse Riogrande II presentaron entre dos y cuatro especies (Staurastrum, Coelastrum y diatomeas). Por tanto, es probable que la dieta de $A$. girodi sea amplia, e involucre más de 10 taxones o especies de algas.

Dentro de las especies de plancton reportadas para el embalse, el $39 \%$ del zooplancton y el $13 \%$ del fitoplancton hicieron parte de la dieta de $A$. girodi. A pesar de que la proporción de taxones para el fitoplancton aparentemente fue menor, durante el análisis de los contenidos estomacales se pudo observar que los estómagos contenían mayor cantidad de componentes del fitoplancton, y además éstos siempre hicieron parte de la dieta. Caso contrario se apreció para el zooplancton que estuvo ausente en muchos estómagos (Tabla 3).

De acuerdo con la frecuencia de ocurrencia de los diferentes ítems alimenticios registrada en los contenidos estomacales de A. girodi, las algas Chlorococcales fueron las más consumidas, ya que representaron el 81\% (Tabla 3). El ítem alimenticio que ocupó el segundo lugar en preferencia fueron las algas pertenecientes al género Ceratium, con alrededor del 32\% de ocurrencia. El tercer y cuarto lugar lo ocuparon los rotíferos $H$. intermedia y $P$. vulgaris con $11 \%$ y $8 \%$ respectivamente, y el quinto lugar correspondió a las algas del género Staurastrum. Por último, el grupo de las diatomeas obtuvo el menor porcentaje de ocurrencia. 
Tabla 2. Medianas de las densidades (ind/I) de A. girodi y de las especies de zooplancton que conformaron su dieta, que presentaron diferencias espaciales estadísticamente significativas (Pruebas Kruskal-Wallis y Mann-Whitney). En negrita los valores correspondientes al sitio diferente. Embalse Riogrande II, agosto 2002 - julio 2003.

\begin{tabular}{|l|c|c|c|c|}
\hline \multicolumn{1}{|c|}{ Taxones } & Chico arriba & Chico abajo & Presa & $\mathbf{X}^{\mathbf{2}}$ \\
\hline Bosmina freyi & $\mathbf{3 1 3 . 7}$ & 110.2 & 116.2 & 14.05 \\
\hline Asplanchna girodi & $\mathbf{1 3 1 . 0}$ & 23.7 & 27.8 & 8.61 \\
\hline Trichocerca similis & $\mathbf{3 6 . 5}$ & 18.0 & 4.3 & 33.97 \\
\hline Polyarthra vulgaris & 32.5 & 6.4 & 1.1 & 24.48 \\
\hline Hexarthra intermedia & $\mathbf{1 7 . 1}$ & 8.7 & 1.4 & 17.61 \\
\hline Filinia longiseta & $\mathbf{0 . 7}$ & 0.9 & 0.0 & 9.69 \\
\hline \multicolumn{2}{|r|}{} & & gl: $32, \mathrm{P}<0.05$ \\
\hline
\end{tabular}

La mayor presencia de fitoplancton en los estómagos analizados indican que $A$. girodi presentó mayor consumo o preferencia por las algas (Figura 2). Adicionalmente, el Índice de Importancia Alimentaria -IIA, mostró la menor relevancia del zooplancton dentro de la dieta de $A$. girodi. En otras palabras, todos los ítems del zooplancton fueron presas de importancia secundaria según el IIA (Tabla 3).

El análisis discriminante de ítems alimenticios totales (fitoplancton y zooplancton) de A. girodi, agrupados por fecha de muestreo arrojó $59.8 \%$ de los casos correctamente clasificados. Se observaron dos funciones

Tabla 3. Ítems alimenticios en estómagos de A. girodi, registrados en tres sitios del embalse Riogrande II. Total de estómagos analizados: 2610. Además, se presenta la frecuencia de ocurrencia de estos ítems y el Índice de Importancia Alimentaria (ÍlA) para

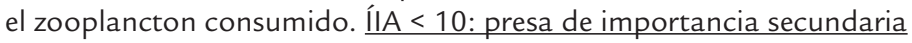

\begin{tabular}{|c|c|c|c|c|c|c|}
\hline $\begin{array}{l}\text { División o } \\
\text { Phylum }\end{array}$ & Orden & Familia & Taxón & $\begin{array}{c}\text { No. de } \\
\text { estómagos }\end{array}$ & $\begin{array}{c}\text { Frecuencia } \\
\text { ocurrencia } \\
(\%)\end{array}$ & $\begin{array}{c}\text { Índice de } \\
\text { Importancia } \\
\text { Alimentaria ÍIA }\end{array}$ \\
\hline \multicolumn{7}{|l|}{ Fitoplancton } \\
\hline Chlorophyta & Sphaeropleales & Scenedesmaceae & Chlorococcales & 2127 & 81.5 & \\
\hline Miozoa & Gonyaulacales & Ceratiaceae & Ceratium & 845 & 32.4 & \\
\hline Charophyta & Desmidiales & Desmidiaceae & Staurastrum & 184 & 7.1 & \\
\hline Chlorophyta & Sphaeropleales & Scenedesmaceae & Coelastrum & 59 & 2.3 & \\
\hline Bacillariophyta & & & diatomeas & 13 & 0.5 & \\
\hline \multicolumn{7}{|l|}{ Zooplancton } \\
\hline Eurotatoria & Floscularicea & Hexarthridae & Hexarthra intermedia & 292 & 11.2 & 3.86 \\
\hline Eurotatoria & Ploima & Synchaetidae & Polyarthra vulgaris & 208 & 8.0 & 2.34 \\
\hline Eurotatoria & Ploima & Brachionidae & Keratella cochlearis & 114 & 4.4 & 0.45 \\
\hline Eurotatoria & Ploima & Trichocercidae & Trichocerca sp. & 79 & 3.0 & 0.44 \\
\hline Eurotatoria & Ploima & Trichocercidae & Trichocerca similis & 52 & 2.0 & 0.20 \\
\hline Branchiopoda & Anomopoda & Bosminidae & Bosmina freyi & 37 & 1.4 & 0.07 \\
\hline Eurotatoria & Floscularicea & Trochosphaeridae & Filinia longiseta & 31 & 1.2 & 0.06 \\
\hline Eurotatoria & Ploima & Synchaetidae & Synchaeta pectinata & 25 & 1.0 & 0.04 \\
\hline Eurotatoria & Ploima & Brachionidae & Keratella americana & 23 & 0.9 & 0.03 \\
\hline Branchiopoda & Anomopoda & Bosminidae & Efipio & 20 & 0.8 & \\
\hline
\end{tabular}


significativas de acuerdo con la prueba de Lambda Wilks $\left(X^{2}=561.02 ;\right.$ g.l. $=420 ; P<0.01$ para la función $1-\mathrm{f} 1 ; \mathrm{X}^{2}=475.176 ; \mathrm{g} . \mathrm{l}=378 ; \mathrm{P}<0.01$ para la función 2 - f2) explicando un porcentaje de la varianza acumulado del $41.5 \%$. Estas funciones están demarcadas principalmente para la f1 por $K$. americana en el eje positivo y por Ceratium en el eje negativo, y para la $\mathrm{f} 2$ por $\mathrm{H}$. intermedia en el eje positivo y por las diatomeas en el eje negativo (Tabla 4).

Tabla 4. Matriz de estructura del análisis discriminante de ítems alimenticios totales de $A$. girodi, en el embalse Riogrande II, agosto 2002 - julio 2003.

\begin{tabular}{|l|c|c|}
\hline \multirow{2}{*}{ Variable } & \multicolumn{2}{c|}{ Función } \\
\cline { 2 - 3 } & $\mathbf{1}$ & $\mathbf{2}$ \\
\hline Hexarthra intermedia & 0.178 & $\mathbf{0 . 5 7 6}$ \\
\hline Ceratium & $\mathbf{- 0 . 3 1 5}$ & 0.082 \\
\hline Coelastrum & -0.255 & 0.325 \\
\hline Bosmina freyi & -0.191 & 0.215 \\
\hline Polyarthra vulgaris & -0.072 & 0.192 \\
\hline Synchaeta pectinata & 0.046 & 0.262 \\
\hline Keratella cochlearis & 0.230 & 0.047 \\
\hline Trichocerca sp & 0.198 & 0.130 \\
\hline Chlorococcales & 0.058 & 0.102 \\
\hline Staurastrum & 0.223 & 0.009 \\
\hline Filinia longiseta & 0.151 & 0.149 \\
\hline Keratella americana & $\mathbf{0 . 3 3 6}$ & -0.023 \\
\hline Trichocerca similis & 0.242 & -0.024 \\
\hline Diatomeas & 0.224 & $\mathbf{- 0 . 0 6 2}$ \\
\hline
\end{tabular}

En la figura 3 se observa la homogeneidad temporal de las muestras formándose un sólo grupo, exceptuando cuatro fechas $(1,5,7$ y 28 , correspondientes a septiembre 19, octubre 30, noviembre 22 de 2002 y julio 21 de 2003, respectivamente). Éstas muestran tendencia positiva en $\mathrm{f} 1$ en las fechas 1,5 y 7 y en f2 en la fecha 28, posiblemente debido a la aparición de K. americana en septiembre 19, octubre 30 y noviembre 22 de 2002; que no se repitió en todo el periodo de estudio, y debido a un pico de aparición superior de $H$. intermedia el 21 de julio de 2003, respecto a los otros muestreos. Aunque se separaron del gran conglomerado, estos centroides no representan una gran diferencia de agrupación con respecto a las otras fechas de muestreo.
Los análisis discriminantes de ítems alimenticios de $A$. girodi agrupados por sitio de muestreo no arrojaron funciones significativas. Sin embargo, dentro de los contenidos estomacales, dos grupos de algas mostraron correlaciones significativas con la variación de la abundancia de este rotífero. Entre ellos el grupo de diatomeas presentó valores de correlación altos y positivos $\left(P<0.05, R^{2}=0.91,0,97\right.$ y 0.87$)$ en los tres sitios de muestreo (Chico arriba, Chico abajo y Presa, respectivamente), y Ceratium mostró una tendencia parecida en los sitios Chico abajo y Presa $\left(P<0.05, R^{2}=\right.$ 0.81 y 0.79 , respectivamente). Estos valores indican un crecimiento conjunto de las poblaciones de A. girodi, diatomeas y Ceratium.

En los tres sitios de muestreo, entre abril y junio de 2003, se registraron picos de crecimiento poblacional de $A$. girodi que coincidieron con incrementos en las densidades de diatomeas y Ceratium, y picos de alta abundancia para el periodo entre enero y marzo en Chico arriba que coincidieron con un incremento de la densidad de Ceratium (Figura 4). Durante todo el año de estudio, la mayoría de las densidades de $A$. girodi reportadas para el sitio Chico arriba superaron las densidades registradas en los otros dos sitios de muestreo, en aproximadamente cinco veces (Figura 4).

En los tres sitios de muestreo se observó crecimiento poblacional simultáneo de $A$. girodi y B. freyi. En Chico arriba coincidieron en noviembre 22 de 2002, enero 23 y en los meses de mayo y junio de 2003; en Chico abajo en los meses de mayo y junio; y para el sitio de Presa para el 12 de noviembre y los meses de mayo y junio. Sin embargo, todas las especies del zooplancton presentaron fluctuaciones a lo largo del tiempo en los tres sitios de muestreo, pero no se evidenciaron relaciones significativas con la presencia de $A$. girodi.

De los nueve taxones del zooplancton que se encontraron en los contenidos estomacales de $A$. girodi, solamente $K$. americana mostró correlación estadísticamente significativa $\left(P<0.05, R^{2}=-0.97\right)$ con la variación de la abundancia de $A$. girodi y únicamente en el sitio Chico abajo. Este valor muestra que mientras una población crece la otra disminuye.

\section{Discusión}

El desarrollo de las comunidades bióticas depende en gran medida de la forma como los factores ambientales se articulen y se distribuyan dentro del ecosistema (González, 1988). Entiéndase entonces, por factores ambientales no solamente el ambiente conformado por los factores abióticos, sino también el estructurado por todas las formas de vida existentes. 


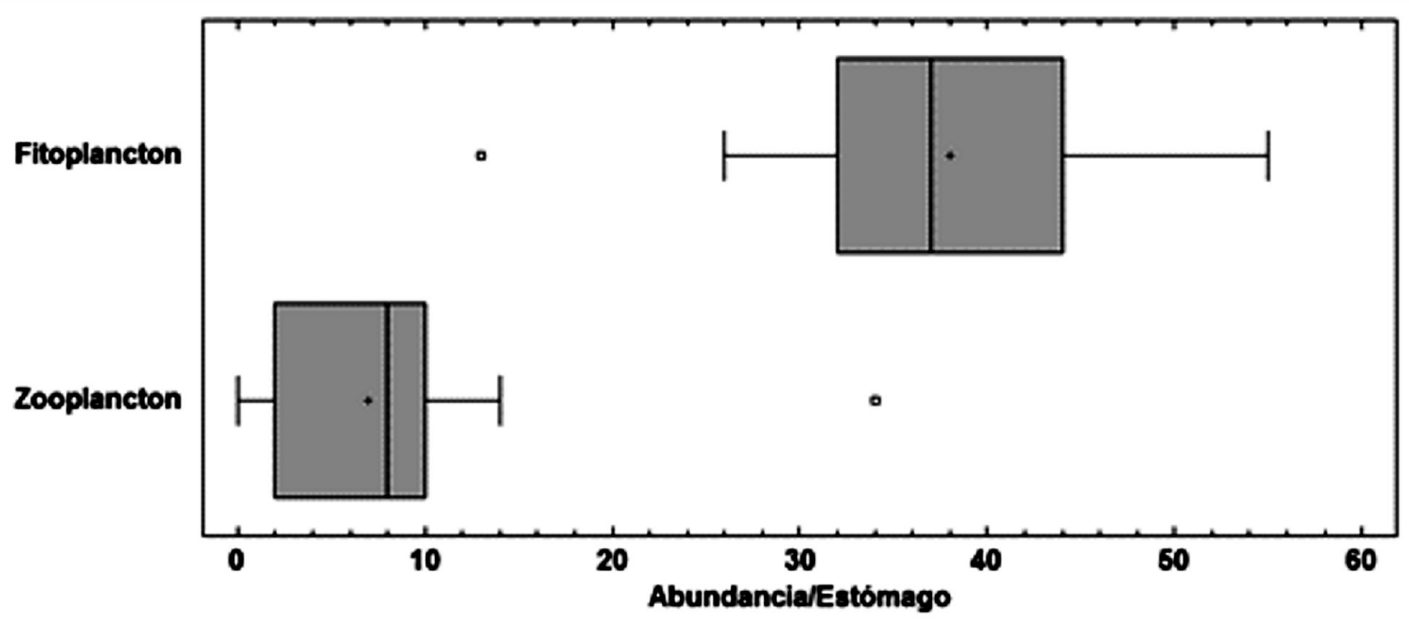

Chico abajo: $P<0.05, X^{2}: 32.80$

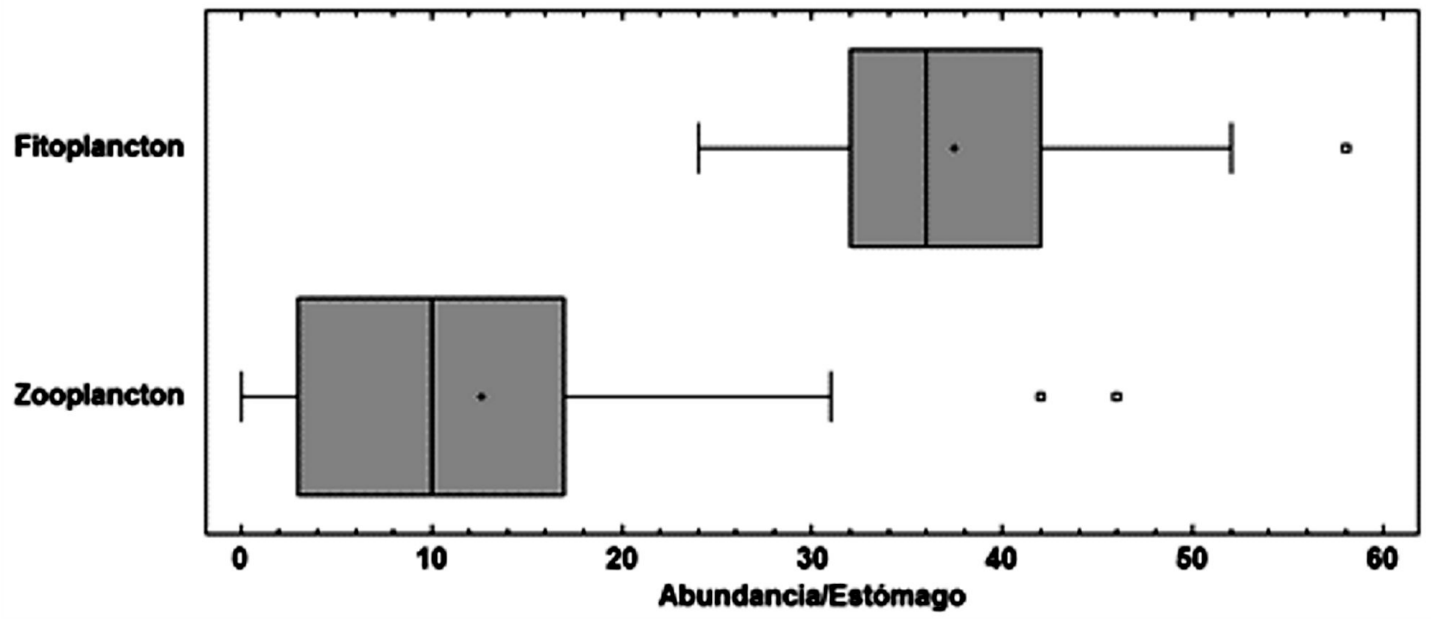

Presa: $P<0.05, X^{2}: 36.3$

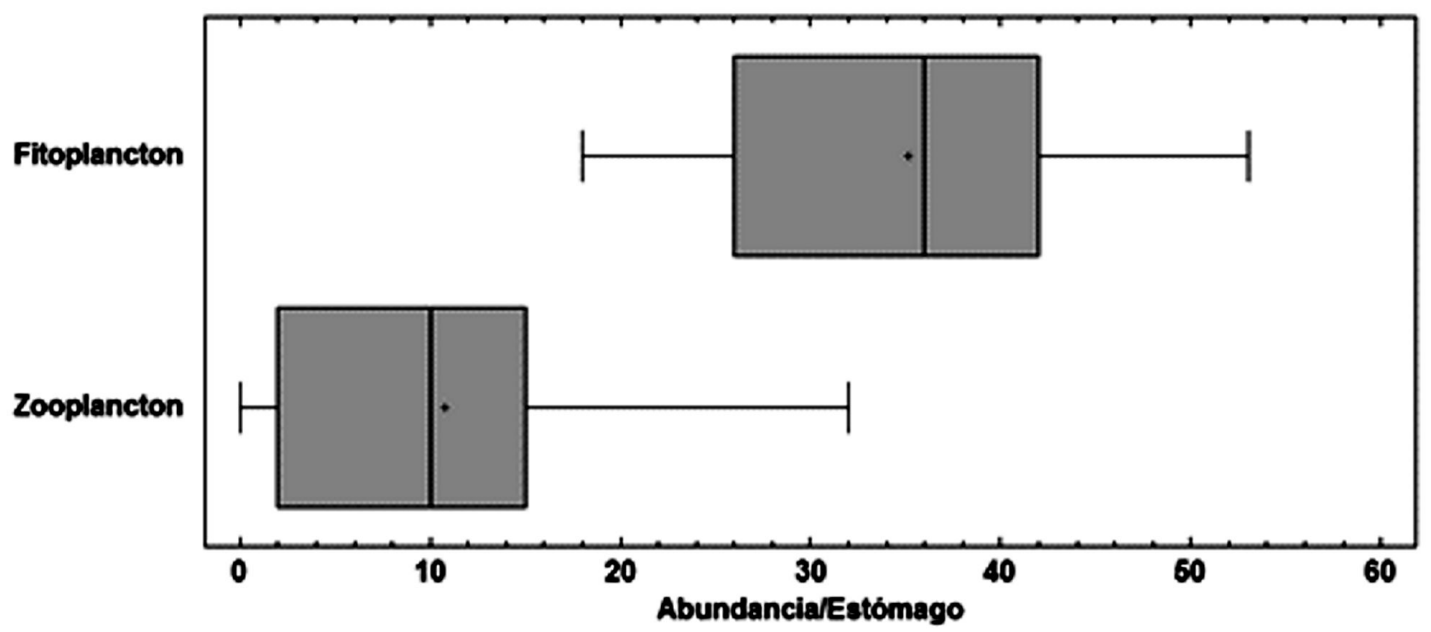

Figura 3. Comparación de la ocurrencia del fitoplancton y el zooplancton en los estómagos de A. girodi, en tres sitios de muestreo del embalse Riogrande II. Agosto 2002 - julio 2003, 


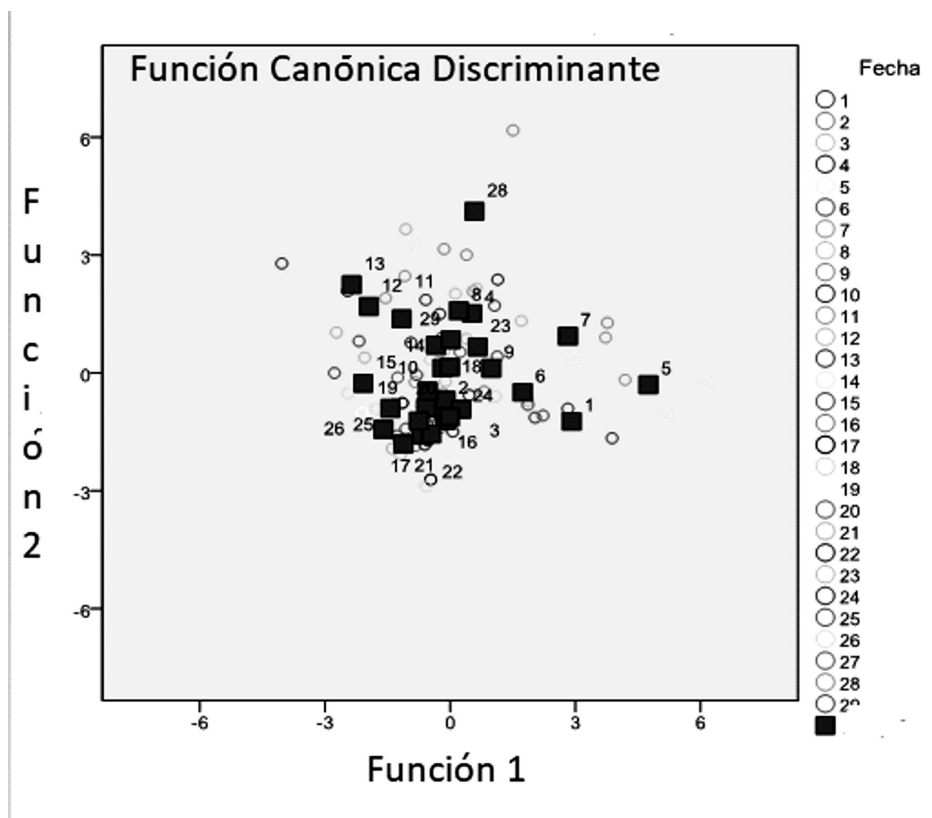

Figura 4. Análisis discriminante de los ítems alimenticios totales de $A$. girodi, agrupados por fecha de muestreo en el embalse Riogrande II. Agosto 2002 - julio 2003.

De acuerdo con las variables abióticas evaluadas se evidenció heterogeneidad espacial en el eje longitudinal sobre el río Chico, con dos sitios altamente contrastantes: Chico arriba y Presa. La ubicación del sitio Chico arriba, cercano a la entrada del material aportado por el río Chico, favorece la alta concentración de sólidos y nutrientes. En contraste, al sitio de Presa llegan cantidades reducidas de los materiales alóctonos, debido a que estos se han sedimentado y metabolizado en los primeros 11 kilómetros de recorrido dentro del embalse (Straškraba, 1999).

La reducida penetración de la luz en el sitio Chico arriba estuvo directamente asociada con mayores cantidades de sólidos aportados por el río afluente, sólidos suspendidos y el fitoplancton. Las características observadas aguas abajo se asociaron posiblemente con la dinámica de sedimentación y asimilación a lo largo del brazo del río Chico, ya que en los primeros tramos de un embalse se depositan los sedimentos de mayor tamaño y se asimila gran parte de los nutrientes entrantes (Straskraba,1999).

Entre los productores primarios, responsables por las concentraciones de biomasa algal en el embalse, se encontró el género Ceratium (Lampert y Sommer 1997, Estrada-Posada 2007), el cual dominó junto con especies del género Staurastrum y del Orden Chlorococcales. Estos taxones son propios de cuerpos de agua estratificados y con una profundidad de mezcla reducida (Lampert y Sommer 1997, Ramírez 2000). En este caso la alta biomasa fitoplanctónica (cuantificada como clorofila a en esta investigación) coincidió con picos de densidades de $A$. girodi.

El consumo de algas ha sido incluido dentro de las estrategias alimenticias de los animales. Sin embargo, existe un sin número de posibles dietas: en un extremo están los generalistas con hábitos que involucran caza, depredación y herbivoría de diferentes ítems alimenticios; mientras en el otro extremo se encuentran los especialistas con dietas restringidas las cuales ignoran alimentos potenciales, y toda la energía se enfoca en la búsqueda de alimentos o presas específicos (Beals, 1999).

Las especies de fito y zooplancton, existentes en el embalse Riogrande II, evidenciaron las diferentes posibilidades tróficas para distintos tipos de comensales; facilitadas, además, por la heterogeneidad del ambiente, donde se observaron múltiples alternativas y hábitos alimenticios, entre ellos la filtración, la herbivoría, la depredación, la succión de contenidos celulares, la invasión, la ruptura de células y la micosis.

En el embalse Riogrande II, la presencia de individuos de algas, rotíferos y cladóceros dentro de los contenidos estomacales de $A$. girodi, indican que posiblemente este rotífero es una especie omnívora, que no ejerce presión de consumo sobre ítems alimenticios específicos. A menudo se ha establecido al género Asplanchna como omnívoro con preferencia por otros rotíferos (King y Snell 1980; Sarma et al., 2003). Sin 


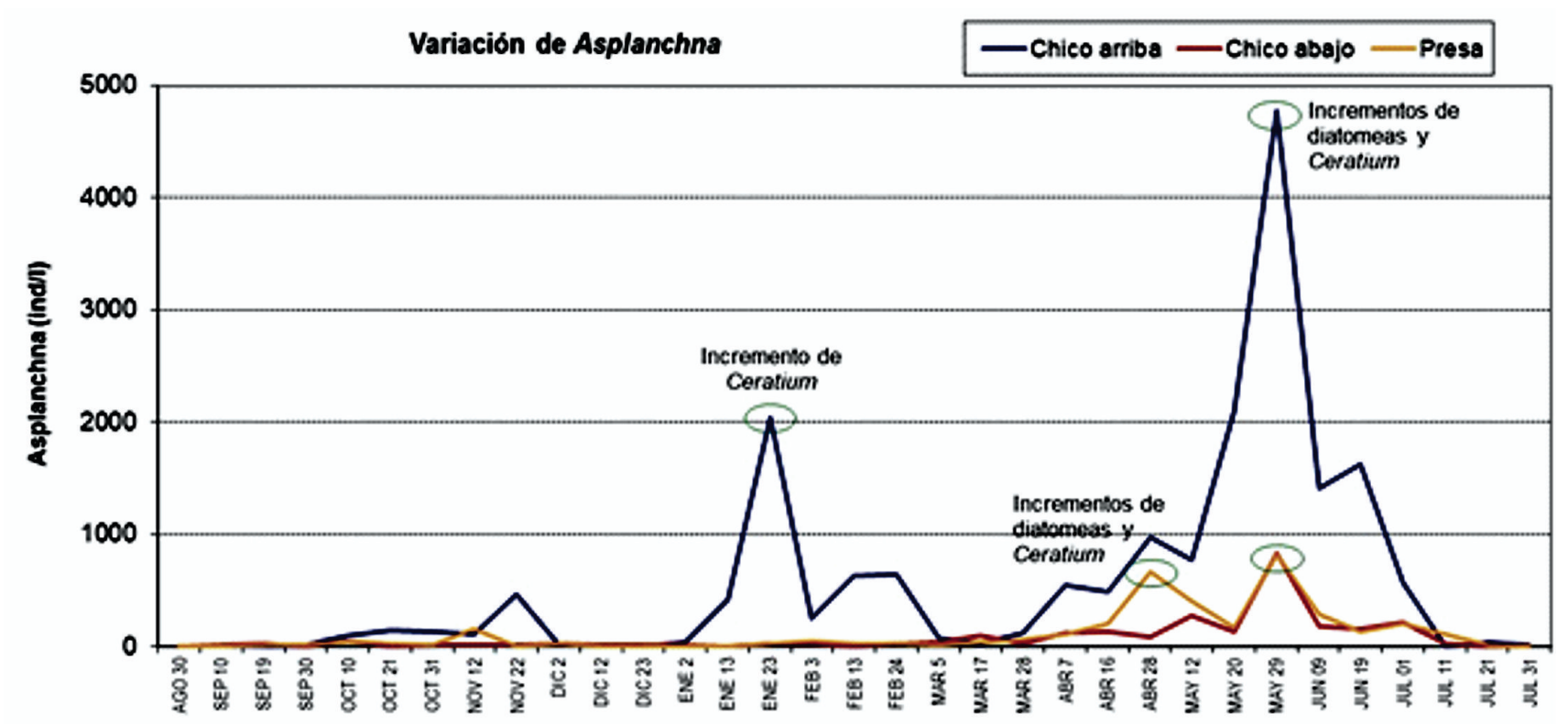

Figura 5. Variación temporal y espacial de la densidad (ind/l) de A. girodi, para el periodo agosto 2002 - julio 2003 en el embalse Riogrande II; indicando las fechas cuando ocurren incrementos en la densidad de diatomeas y de Ceratium.

embargo, se ha demostrado que especies de este género pueden alimentarse principalmente de algas en el caso de A. priodonta y A. herricki (Chang et al., 2010), sólo de individuos algales como Chlorella minutissima en cultivos de A. silvestrii (Hampton y Starkweather 1998), de protistas como Paramecium caudatum en cultivos de A. brightwelli (Wang-Yin y Juan-Niu 2008) o de microcrustáceos como Artemia franciscana en cultivos de A. silvestrii (Hampton 1998).

En el caso específico de A. girodi, Chang et al., (2010) la han catalogado como una especie depredadora, pues su dieta estuvo compuesta principalmente de especies zooplanctónicas. Sin embargo,en el presente estudio se buscó establecer la dieta real de A. girodi, lo que sólo es posible mediante el análisis de los contenidos estomacales de individuos colectados directamente en el medio natural. Este procedimiento permitió encontrar que la mayor proporción de los ítems alimenticios que conformaron la dieta de este rotífero fueron de origen algal; específicamente dirigida al consumo de Chlorococcales y Ceratium, presentes en el 81 y $32 \%$ de los estómagos analizados, respectivamente.

En contraste, el consumo de zooplancton estuvo representado en proporción baja, registrándose como máximo en el $11 \%$ de los estómagos analizados $(H$. intermedia). Lo anterior indica que existió una marcada preferencia de $A$. girodi por el consumo de algas en el embalse Riogrande II y que podría ser catalogada como especie omnívora, con una dieta basada principalmente en algas; aunque algunos autores conside- ran que la nutrición zoófaga es ventajosa por las altas tasas de asimilación (Kappes et al., 2000).

La coincidencia en los picos de abundancia de A. girodi con las épocas de incremento en la densidad de las algas, indican que este rotífero prefiere consumir y aprovechar los ítems con mayor disponibilidad, en este caso las algas del fitoplancton. Preferencias de este tipo también se reportan para $A$. priodonta dentro del lago Windsborn en Alemania, que además fue clasificada como forrajera oportunista y no como depredadora (Kappes et al., 2000). Su dieta inclúa altas proporciones de algas coloniales y cianobacterias; y en contraste el contenido zooplanctónico dentro de su estómago fue raramente encontrado. La plasticidad de este rotífero ha sido demostrada, variando de poblaciones zoófagas, a omnívoras y a exclusivamente fitófagas (Kappes et al., 2000).

A partir de los resultados obtenidos para el embalse Riogrande II, considerando el amplio espectro de muestreos (36 fechas a lo largo de un año y tres sitios contrastantes) y que se analizaron 2610 contenidos estomacales, se concluye que $A$. girodi presentó el mismo comportamiento trófico de A. priodonta (Kappes et al., 2000); con consumo preferente por algas, pero manteniendo depredación de rotíferos y bosmínidos en una proporción menor (aproximadamente del $10 \%$ ). La preferencia por uno u otro alimento podría ser vista desde dos contextos diferentes: El primero implicaría la inclinación por alimentos considerados más "valiosos" energéticamente o por aquellos ítems 
alimenticios que hacen parte integral de una dieta balanceada; o el segundo involucraría dietas oportunistas o mixtas que podrían llevar a ingerir ítems alimenticios de "baja calidad" por el simple hecho de habérselos encontrado (Begon et al., 2006).

Sin embargo, las preferencias de muchos consumidores pueden estar dirigidas a consumir aquellos ítems cuya abundancia sea mayor. La situación más común se presenta cuando diferentes tipos de ítems alimenticios se encuentran en distintos microhábitats, y los consumidores se concentran en estos, como es el caso de las mayores abundancias encontradas en Chico arriba para A. girodi, donde la biomasa del fitoplancton era cinco veces mayor. No obstante, ningún organismo es capaz de consumir todos los tipos de ítems alimenticios, y el número de éstos es limitado de acuerdo con sus características morfológicas. La amplitud de la dieta, en relación con la variedad y el posible potencial de alimentación de un organismo, se explica mediante la teoría del forrajeo óptimo.

Cuando el forrajeador óptimo expande su dieta, disminuye su tiempo de búsqueda e incrementa la rentabilidad, dado que reduce la energía empleada para encontrar, manipular y consumir su alimento. Un forrajeador especialista busca un tipo específico de presa hasta encontrarla y ésta le otorga una cantidad de energía proporcional a su esfuerzo. Como generalista el comensal emplea una mayor diversidad de presas que encuentra con mayor facilidad, invirtiendo poco tiempo y energía (Begon et al., 2006). Así mismo, la diversidad de presas puede en muchos casos suplir las carencias ocasionadas por una dieta oportunista.

Los datos de abundancia de los contenidos estomacales de zooplancton dentro de $A$. girodi fueron bajos y altamente variables, pero al no mostrar diferencias estadísticas espacial y temporalmente se concluye que la población de este rotífero se alimentó sin un patrón definido para los ítems zooplanctónicos durante el periodo de estudio. En relación con el fitoplancton, las explosiones poblacionales de $A$. girodi se presentaron luego de picos de abundancia de Ceratium y diatomeas en todos los sitios de muestreo. Esto podría revelar que mientras estos taxones incrementan su densidad, A. girodi también lo hace; indicando oportunismo trófico de un recurso dentro del sistema que antes no estaba disponible. Podría indicarse una correlación directa de Ceratium como recurso y $A$. girodi como consumidor, hecho que se comprueba cuando se observa que estos dos taxones (alga y rotífero) incrementan sus densidades simultáneamente $(\mathrm{r}=0.79, \mathrm{P}<0.01)$ (Estrada-Posada, 2007); sin embargo, la presión de consumo de este rotífero sobre Ceratium es mínima.
A partir de los resultados obtenidos en este estudio, se puede postular que $A$. girodi no exhibe respuestas funcionales ni numéricas a la densidad de la mayoría de sus presas y, por tanto, probablemente esta especie no tiene un efecto regulatorio importante en el plancton de aguas continentales. Los datos de densidad indican que el plancton del embalse Riogrande II no es susceptible al consumo de $A$. girodi, exceptuando a $K$. americana, para la cual aún no se ha logrado establecer un patrón en campo, debido a su baja frecuencia de aparición. En otras palabras las preferencias alimentarias de $A$. girodi no constituyen un factor de presión en la dinámica poblacional del fitoplancton ni del zooplancton; sin embargo se logra apreciar una relación directa en el crecimiento poblacional de este rotífero y algunas de las algas que constituyen su dieta.

Por último y en relación con las hipótesis planteadas se concluye que:

1. Se acepta la hipótesis, pues $A$. girodi es una especie omnívora, con mayor preferencia por el consumo de algas.

2. Este rotífero no fue factor de presión en la dinámica poblacional de las algas y zooplancton en general, pero posiblemente lo fue para el rotífero K. americana, por lo que esta hipótesis se acepta parcialmente.

3. Se acepta parcialmente, pues no se observaron diferencias espaciales en la dieta de A. girodi, pero si existieron diferencias temporales en cuanto a la composición de la dieta, la cual estuvo determinada por la oferta de las algas, principalmente durante florecimientos de Ceratium.

Se concluye que la dieta de $A$. girodi se enmarca dentro de los conceptos de forrajeo óptimo y oportunismo trófico, estrategias que conllevan el consumo de las especies de mayor disponibilidad (en este caso algas) y el máximo ahorro de energía, puesto que no se invierte en la búsqueda de presas específicas (Begon et al., 2006, Da Silva y Hahn, 2009).

\section{Agradecimientos}

A los funcionarios del embalse Riogrande II y de las Empresas Públicas de Medellín que hicieron posible las jornadas para toma de las muestras. Al profesor John Jairo Ramírez Restrepo, por facilitar el espacio y los equipos necesarios para realizar el análisis de los contenidos estomacales de los individuos de A. girodi. Al equipo humano y académico del Laboratorio de Limnología del grupo LimnoBasE BiotaMar, del Instituto de Biología de la Universidad de Antioquia, en cuya 
colección biológica se mantuvieron las muestras de plancton colectadas por Estrada-Posada (2007).

\section{Referencias}

Beals M, Gross L, Harrell S. 1999. Optimal Foraging Theory. (Febrero 2019). URL: http://www.tiem.utk.edu/ gross/bioed/bealsmodules/optimal.html

Begon M, Townsend CR, Harper JL. 2006. Ecology: from individuals to ecosystems. Fourth Edition. Blackwell Publishing. Oxford, Inglaterra, p. 752.

Bicudo CEM, Bicudo RMT. 1970. Algas de águas continentais brasileiras. In Algas de águas continentais brasileiras. Funbec.

Chang $\mathrm{KH}$, Doi $\mathrm{H}$, Nishibe $\mathrm{Y}$, Nakano SI. Feeding habits of omnivorous Asplanchna: comparison of diet composition among Asplanchna herricki, A. priodonta and A. girodi in pond ecosystems. J Limnol. 2010;69(29):2009-2016.

Conde-Porcuna JM, Morales-Baquero R, Cruz-Pizarro L. Effectiveness of the caudal spine as a defense mechanism in Keratella cochlearis. Hydrobiologia. 1993;255/256:283-287.

Da Silva MR, Hahn NS. Influência da dieta sobre a abundância de Mopenkhausia dichroura (Characiformes, Characidae) no reservatório de Manso, Estado de Mato Grosso. Iheringia, Sér. Zool., Porto Alegre. 2009;99(3):324-328.

Edmonson WT. 1965. Freshwater Biology. 2a Ed. John Willey \& Sons, Inc. New York. E.U.A. p. 1248.

Elmoor-Loureiro LMA, Mendonça-Galvão L, Padovesi-Fonseca C. New cladoceran records from lake Paranoa, Central Brazil. Braz J Biol. 2004;64(3A):415-422.

EPM. Cincuenta años de hidrometeorología en Empresas Públicas de Medellín. Revista Hidrometeorológica. 2005;1(1):1-150.

Estrada-Posada AL. 2007. Variación de la estructura y la distribución del zooplancton en función de gradientes longitudinales abióticos y bióticos en el embalse Riogrande II (Antioquia), Colombia. [Tesis de Doctorado]. Instituto de Biología. Universidad de Antioquia. Medellín. Colombia, p. 95.

Fernando $\mathrm{CH}$. 2002. A guide to tropical freshwater zooplankton: identification, ecology and impact on fisheries. Backhuys Publishers, Leiden, The Netherlands, p. 300.

Gaviria S. Los copépodos (Arthropoda, Crustacea) de vida libre de las aguas continentales de Colombia. Rev Acad Colomb Cienc Exactas Fis Nat. 1994;19(73):361-385.

Gilbert JJ, Jack JD. Rotifers as predators on small ciliates. Hydrobiologia. 1993; 255(1):247-253.

Giraldo MMA. 2010. Composición de la dieta del rotífero Asplanchna girodi (De Guerne, 1888) y su variación temporal y espacial en el embalse Riogrande II (Antioquia, Colombia). [Trabajo de pregrado]. [Medellín (Colombia)]: Instituto de Biología, Universidad de Antioquia, p. 80.

González IA. 1998. El plancton de las aguas continentales. Monografía No. 33, Serie Biología. Secretaria General de la OEA. Washington, EUA, p. 130.
Hampton SE. 1998. Morphotype-specific predation in the trimorphic rotifer Asplanchna silvestrii. En Rotifera VIII: A comparative approach. Springer, Dordrecht, p. 437-444.

Hampton SE, Starkweather PL. Differences in predation among morphotypes of the rotifer Asplanchna silvestrii. Freshw Biol. 1998;40(4):595-605.

Haney JF, Hall DJ. Sugar-coated Daphnia: A preservation technique for Cladocera. Limnol Oceanogr. 1973;18:331-333.

Hyslop EJ. Stomach content analysis - A review of methods and applications. J Fish Biol. 1980;17:411-429.

Jena M, Adhikary SP. Chlorococcales (Chlorophyceae) of eastern and north-eastern states of India. Algae. 2007;22(3):167-183

Kappes H, Mechenich C, Sinsch U. Long-term dynamics of Asplanchna priodonta in LakeWindsborn with comments on the diet. Hydrobiologia. 2000;432:91-100.

King C, Snell T. Density-dependent sexual reproduction in natural populations of the rotifer Asplanchna girodi. Hydrobiologia. 1980;73:149-152.

Koste W, Shield RJ. Rotifera from Australian Inland. Waters II. Epiphanidae and Brachionidae (Rotifera: Monogonta). Invertebrate Taxonomy. 1987; 1(7):949-1021

Koste W. 1978. Rotatoria. Die Rädertiere Mitteleuropas. Überordnung Monogononta. Gebruder Borntrager. Berlin, Alemania. p. 673 .

Lampert W, Sommer U. 1997. Limnoecology: The Ecology of Lakes and Streams. Oxford University Press, New York. p. 382.

Lauzanne L. Régime alimentaire d'Hidrocyon forskali (Pisces: Characidae) dans le lac Tchad et ses tributaires. Cahiers ORSTOM, Série Hydrobiologie. 1975;9(2): 105-121.

Mazo BD, Ramírez-Restrepo JJ, Díaz-C A. Caracterización física y química del embalse Riogrande II (Antioquia), Colombia. Actualidades Biológicas. 2015;37(103):155-168.

Paggi JC. 1995. Crustacea Cladocera. En: Lopretto, E. \& Tell, G. (eds.) Ecosistemas de aguas continentales: Metodología para su estudio. La Plata, Argentina: Ediciones Sur. p. 909-951.

Pennak RW. 1989. Freshwater invertebrates of the United States. 3a ed. John Willey y Sons, Inc. New York, E.U.A. p. 803.

Prescott GW. 1962. Algae of the western Great Lakes area. 2nd ed. Wm. C. C. Brown Co. Dubuque, lowa. EUA. Vol 946.

Ramírez JJ. 2000. Fitoplancton de Agua Dulce: Aspectos Ecológicos, Taxonómicos y Sanitarios. Editorial Universidad de Antioquia Medellín, Colombia. p. 207.

Reid JW. Chave de identificação e lista de referências bibliográficas para as espécies continentais sulamericanas de vida livre da ordem Cyclopoida (Crustacea, Copepoda). Boletim de Zoologia. 1985;9(9):17-143.

Rocha O, Tundisi M. 1976. Atlas do zooplâncton (Represa de Broa, São Carlos). Vol. I. Copepoda. Universidade Federal de São Carlos. São Carlos, Brasil. p. 153. 
Sarma SSS, Dumont HJ. Demography and population growth of Asplanchna girodi (Rotifera) as a function of prey (Anuraeopsis fissa) density. Hydrobiologia. 1995;306:97-107.

Sarma SSS, Nandini S. Small prey size offers immunity to predation: a case study of two species of Asplanchna and three brachionid prey (Rotifera). Hydrobiologia. 2007;593:67-76.

Sarma SSS, Nandini S, Dumont HJ. Feeding preference and population growth of Asplanchna brightwelli (Rotifera) offered two nonevasive prey rotifers. Hydrobiologia. 1998;361:77-87.

Sarma SSS, Pavon-Mezal EL, Nandini S. Comparative population growth and life table demography of the rotifer Asplanchna girodi at different prey (Brachionus calyciflorus and Brachionus havanaensis) (Rotifera) densities. Hydrobiologia. 2003;491:309320.

Sartory DP, Grobbelaar JU. Extraction of chlorophyll a from freshwater phytoplankton for spectrophotometric analysis. Hydrobiologia. 1984;114:177-187.

Segers H. A biogeographical analysis of rotifers of the genus Trichocerca Lamarck, 1801 (Trichocercidae, Monogononta, Rotifera), with notes on taxonomy. Hydrobiologia. 2003;500:103-114.
Sendacz S, Kubo E. Copepoda (Calanoida e Cyclopoida) de reservatórios do Estado de São Paulo. Boletim do Instituto de Pesca (São Paulo), 1982;9:51-89.

Shield RJ, Koste W. Rotifera from Australian inland waters VIII. Trichocercidae (Monogononta). T Roy Soc South Aust. 1992;116(1):1-27.

Shield RJ, Koste W. Rotifera from Australian inland waters. IX. Gastropodidae, Synchaetidae, Asplanchnidae (Rotifera: Monogononta). T Roy Soc South Aust. 1993;117(3):111-139.

Straškraba M. 1999. Retention time as a key variable of reservoir limnology. p. 385-410. En: Tundisi JG, Straskraba M. 1999. Theoretical reservoir ecology and its applications. Brazilian Academy of Sciences and Backhuys Publishers. São Carlos, Brasil.

Van der Stap I, Vos M, Mooij WM. Inducible defenses and rotifer food chain dynamics. Hydrobiologia. 2007;593:103-110.

Wang-Ying X, Juan-Niu C. Predatory rotifer Asplanchna brightwellii mediated competition outcome between Brachionus calyciflorus and Brachionus patulus (Rotifera). Hydrobiologia. 2008;610:131-138. 